\title{
Niaspan inhibits diabetic retinopathy-induced vascular inflammation by downregulating the tumor necrosis factor- $\alpha$ pathway
}

\author{
YANG WANG, XIANGDA MENG and HUA YAN \\ Department of Ophthalmology, Tianjin Medical University General Hospital, Tianjin 300052, P.R. China
}

Received November 25, 2015; Accepted November 22, 2016

DOI: $10.3892 / \mathrm{mmr} .2017 .6146$

\begin{abstract}
Diabetic retinopathy (DR) is a serious microvascular complication of diabetes and a major cause of blindness in the developing world. Early DR is characterized by vascular neuroinflammation, cell apoptosis and breakdown of the blood-retinal barrier (BRB). However, optimal treatment options and associated mechanisms remain unclear. Niaspan, which is widely used in the prevention and treatment of hyperlipidemia-associated diseases, has been reported to inhibit inflammation. However, the effects of Niaspan and the mechanisms underlying the anti-inflammatory effects of Niaspan on DR have yet to be reported. The present study aimed to investigate the anti-inflammatory effects and mechanisms of Niaspan in a rat model of DR. Rats with DR exhibited a significant increase in BRB breakdown, retinal apoptosis, and tumor necrosis factor- $\alpha$ (TNF- $\alpha$ ) and nuclear factor- $\kappa B$ $(\mathrm{NF}-\mathrm{\kappa B})$ expression. In addition, the expression levels of inducible nitric oxide synthase (iNOS) and intercellular cell adhesion molecule-1 (ICAM-1) were increased in the retinas of DR rats compared with in the normal control group. In conclusion, treatment with Niaspan significantly improved clinical and histopathological outcomes; decreased the expression levels of TNF- $\alpha$, NF- $\kappa$ B, iNOS and ICAM-1; and decreased apoptosis and BRB breakdown, as compared with in the retinas of DR rats. The present study is the first, to the best of our knowledge, to demonstrate that Niaspan treatment ameliorates DR by inhibiting inflammation, and also suggests
\end{abstract}

Correspondence to: Dr Hua Yan, Department of Ophthalmology, Tianjin Medical University General Hospital, 154 Anshan Road, Tianjin 300052, P.R. China

E-mail: phuayan2000@163.com

Abbreviations: DR, diabetic retinopathy; TNF- $\alpha$, tumor necrosis factor- $\alpha$; NF- $\kappa \mathrm{B}$, nuclear factor- $\kappa \mathrm{B}$; STZ, streptozotocin; BRB, blood-retinal barrier; ICAM-1, intercellular cell adhesion molecule-1; iNOS, inducible nitric oxide synthase; $\mathrm{H} \& \mathrm{E}$, hematoxylin and eosin; GCL, ganglion cell layer; INL, inner nuclear layer; ONL, outer nuclear layer

Key words: diabetic retinopathy, Niaspan, vascular inflammation, TNF- $\alpha$ pathway, blood-retinal barrier, apoptosis that the TNF- $\alpha$ pathway may contribute to the beneficial effects of Niaspan treatment.

\section{Introduction}

Diabetic retinopathy (DR) is a serious microvascular complication of diabetes and a major cause of blindness, which usually affects individuals between 30 and 70 years old (1). In a previous study, neuroinflammation has been suggested as an early event in the pathogenesis of DR (2). Diabetes affects the entire neurovascular unit of the retina, with gradual neurodegeneration, gliosis, neuroinflammation, vascular abnormalities including plasma leakage, compromised vascular blood-retinal barrier (BRB), edema, angiogenesis, and eventual fibrosis, all of which occur at increasing frequency (3). However, the treatment options for DR remain limited and are often associated with adverse effects; therefore, patients with diabetes have a high risk of eventual blindness. There is an emerging requirement to develop novel therapeutic approaches for this devastating disease.

Niacin (vitamin B3 or nicotinic acid) is the most effective medication for the treatment of atherosclerosis in current clinical use, which increases high-density lipoprotein levels, and substantially lowers total cholesterol and triglyceride levels (4). Niaspan is a prolonged release formulation of niacin, which is safe to use in patients with diabetes (5). It has previously been reported that prolonged niacin treatment may exert anti-inflammatory effects (6). Furthermore, niacin has been revealed to inhibit vascular inflammation by downregulating the nuclear factor- $\kappa \mathrm{B}(\mathrm{NF}-\kappa \mathrm{B})$ signaling pathway (7). However, the anti-inflammatory effects of Niaspan on DR have yet to be elucidated.

The present study aimed to examine the anti-inflammatory effects of Niaspan on streptozotocin (STZ)-induced DR. The results demonstrated that administration of Niaspan .3 months after the induction of diabetes significantly improved functional outcome, and inhibited vascular inflammation in the retina.

\section{Materials and methods}

Animals. Adult Male Wistar rats (age, 7 weeks; weight, 225-250 g) were purchased from the Academy of Military Medical Science (Beijing, China). The rats were housed in specific pathogen-free conditions (temperature $22 \pm 2{ }^{\circ} \mathrm{C}$, 
light/dark cycle 12/12 h) with ad libitum access to food and water. All procedures involving rats were approved by the Laboratory Animal Care and Use Committee of Tianjin Medical University (Tinajin, China), and conformed to the Association for Research in Vision and Ophthalmology Statement for the Use of Animals in Ophthalmic and Vision Research (8).

Diabetes induction and treatment. Diabetes was induced via injection of STZ (45 mg/kg; Sigma-Aldrich; Merck Millipore, Darmstadt, Germany) into the tail vein of Wistar rats. Fasting blood glucose levels were determined using a glucose analyzer 6 days after STZ injection; rats with fasting blood glucose levels $>16.7 \mathrm{mmol} / 1$ were identified as diabetic and were used in the present study (9). Niaspan (China Resources Pharmaceutical Group Co., Ltd., Beijing, China) was dissolved in water, and $40 \mathrm{mg} / \mathrm{kg} /$ day was administered following STZ injection (the 7th day following STZ injection). A total of 90 rats were divided into the following groups: i) Normal control group (control group; n=30); ii) DR model group without Niaspan treatment (DR group; $n=30$ ); and iii) DR model group treated with Niaspan (Niaspan group; $n=30$ ).

Histological and immunohistochemical analyses. Rats were anesthetized via injection of chloral hydrate (concentration:10\%; $600 \mathrm{mg} / \mathrm{kg}$ ) into the tail vein of Wistar rats in the third month following Niaspan treatment. Then the eyes were removed and were fixed in $4 \%$ paraformaldehyde with phosphate-buffered saline (PBS; $\mathrm{pH} 7.4$ ) for $2 \mathrm{~h}$ at $4^{\circ} \mathrm{C}$. The eyes were then dehydrated in a graded alcohol series and embedded in paraffin. The paraffin-embedded tissues were cut into $5 \mu \mathrm{m}$ sections. Subsequently, the sections were stained with hematoxylin and eosin (H\&E) by fluorescence microscope (Leica DMI4000B; Leica Microsystems GmbH, Düren, Germany). For immunohistochemical analysis, sections $(5 \mu \mathrm{m})$ were prepared from paraffin-embedded tissues and were incubated overnight at $4^{\circ} \mathrm{C}$ with antibodies against tumor necrosis factor- $\alpha$ (TNF- $\alpha$; polyclonal rabbit anti-rat; cat. no. 74120; 1:100; GeneTex, Inc., Irvine, CA, USA). The sections were then stained with biotinylated anti-rabbit immunoglobulin $\mathrm{G}$ secondary antibody (cat. no. BA-1000; 1:200; Vector Laboratories, Inc., Burlingame, CA, USA) for $2 \mathrm{~h}$ (room temperature) followed by incubation with horseradish peroxidase streptavidin (cat. no. SA-5704; Vector Laboratories, Inc.) for $1 \mathrm{~h}$ (room temperature). Specific labeling was visualized by incubation with diaminobenzidine (DAB; cat. no. ZLI-9017; Zhongshan Golden Bridge Biotechnology Co., Ltd., Beijing, China). Finally, the sections were counterstained with hematoxylin (cat. no. G1080; Solarbio Science \& Technology Co., Ltd., Beijing, China). Images were captured using a Leica DMI4000B (Leica Microsystems GmbH, Wetzlar, Germany) and the results were quantified using Image-Pro Plus 6.0 (Media Cybernetics, Inc., Rockville, MD, USA). Retinal cell numbers in the ganglion cell layer (GCL) were counted in the region within a fixed $100-\mu$ m column.

Western blotting. Western blotting was performed using standard methods. Retinal protein was extracted using a radioimmunoprecipitation assay buffer (Beijing Zhongshan Golden Bridge Biotechnology; OriGene Technologies, Inc., Rockville, MD, USA) and were quantified using a protein assay (Bradford Protein Assay; Bio-Rad Laboratories, Inc., Hercules, CA, USA).
Equal amounts of protein $(800 \mu \mathrm{mol} / \mathrm{l})$ were separated by $8-12 \%$ sodium dodecyl sulfate-polyacrylamide gel electrophoresis and electroblotted onto polyvinylidene fluoride membranes (EMD Millipore, Billerica, MA, USA). The membranes were blocked in 5\% skim milk for $2 \mathrm{~h}$ (room temperature) and were incubated with antibodies against TNF- $\alpha$ (polyclonal rabbit anti-rat; cat. no. 74120; 1:1,000; GeneTex, Inc.), NF-кB (polyclonal rabbit anti-rat; cat. no. 54672; 1:1,000; GeneTex, Inc.), inducible nitric oxide synthase (iNOS; polyclonal rabbit anti-rat; cat. no. ab15323; 1:500; Abcam, Cambridge, UK) and intercellular adhesion molecule-1 (ICAM-1; polyclonal rabbit anti-rat; cat. no. 16174-1-AP; 1:1,000; Proteintech Group, Inc., Rosemont, IL, USA) overnight at $4^{\circ} \mathrm{C}$. Subsequently, the membranes were washed in $0.1 \%$ TBS-Tween-20 and incubated with anti-rabbit IgG secondary antibody (cat. no. ZDR-5306; 1:5,000, Beijing Zhongshan Golden Bridge Biotechnology; OriGene Technologies, Inc.) at room temperature for $1 \mathrm{~h}$. Monoclonal mouse anti- $\beta$-actin (cat. no. TA-09; 1:1,000; Zhongshan Golden Bridge Biotechnology Co., Ltd.) was used as an internal reference. Finally, the blots were scanned with a ChemiDoc ${ }^{\mathrm{TM}}$ MP system (Bio-Rad Laboratories, Inc.) and the bands were semi-quantified using ImageJ 1.51 software (National Institutes of Health, Bethesda, MA, USA).

$R N A$ extraction and reverse transcription-quantitative polymerase chain reaction ( $R T-q P C R)$. Total RNA was isolated from retinas (taken from 3 rats for each group) using TRIzol ${ }^{\circledR}$ reagent (cat. no. 15596; Thermo Fisher Scientific, Inc., Waltham, MA, USA), RNA was reverse transcribed into cDNA using the TransScript First-Strand cDNA Synthesis SuperMix (cat. no. AT301; TransGen Biotech Co., Ltd., Beijing, China). The primer sequences were as follows: $\beta$-actin, forward 5'-AGC CATGTACGTAGCCATCC-3', reverse 5'-ACCCTCATAGAT GGGCACAG-3'; NF-кB, forward 5'-TGAGGCTGTTTGGTT TGAGA-3', reverse 5'-TTATGGCTGAGGTCTGGTCTG-3'; iNOS, forward 5'-TATCTGCAGACACATACTTTACGC-3', reverse 5'-TCCTGGAACCACTCGTACTTG-3'; and ICAM-1, forward 5'-GGCCTCAGTCAGTGTGA-3' and reverse 5'-AAC CCCATTCAGCGTCA-3'. The relative mRNA expression levels of NF- $\kappa \mathrm{B}$, iNOS and ICAM-1 were detected by RT-qPCR with TransStart Top Green qPCR SuperMix (cat. no. AQ131; TransGen Biotech Co., Ltd.). $\beta$-actin mRNA was used as an internal control. All procedures were performed according to the manufacturers' protocols. The relative mRNA expression levels were determined using the $2^{-\Delta \Delta \mathrm{Cq}}$ method (10).

Measurement of BRB breakdown using Evans blue. 2\% Evans blue dye (Sigma-Aldrich; Merck Millipore) in saline was administered via the tail vein of rats ( $n=4 /$ group) as a BRB permeability tracer $2 \mathrm{~h}$ prior to sacrifice. Rats were sacrificed via injection of chloral hydrate (concentration: $10 \% ; 600 \mathrm{mg} / \mathrm{kg}$ ) into the tail vein. for $10 \mathrm{sec}$ at a dose of $45 \mathrm{mg} / \mathrm{kg}$, and after the dye had circulated for $120 \mathrm{~min}$, the eyes were immediately fixed in $4 \%$ paraformaldehyde for $2 \mathrm{~h}$. Subsequently, the anterior segments were removed and the retinas were dissected and washed in cold PBS. The retinas were then spread on glass slides, vitreous side up, and mounted with mounting medium. Images were captured using a confocal scanning laser imaging system fitted with krypton-argon lasers (FV1000; Olympus Corporation, Tokyo, Japan). 
A

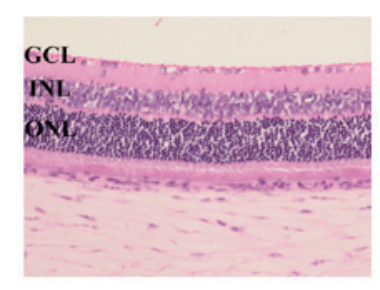

B
DR

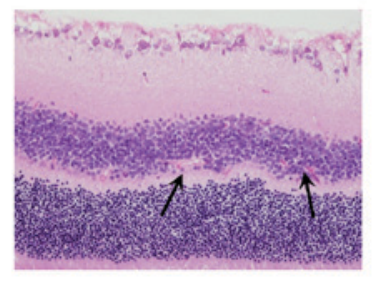

NA

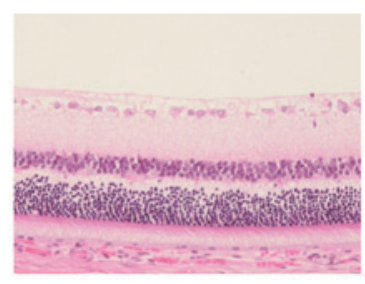

Retinal cell counts

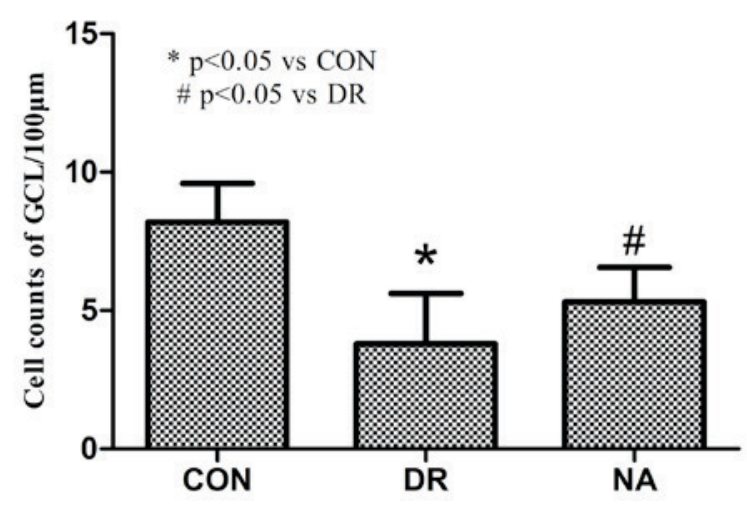

Figure 1. H\&E staining of the retina in a DR model. (A) H\&E staining of the retina (n=7/group). (B) Quantitative analysis of retinal cell counts in the GCL. The arrows indicate hemorrhage in the INL. ${ }^{*} \mathrm{P}<0.05$ compared with nondiabetic retina in the CON group; ${ }^{*} \mathrm{P}<0.05$ compared with diabetic retina in the $\mathrm{DR}$ group. CON, control; DR, diabetic retinopathy; NA, Niaspan; H\&E, hematoxylin and eosin; GCL, ganglion cell layer; INL, inner nuclear layer; ONL, outer nuclear layer.

Quantitative evaluation of Evans blue dye extravasation. Evans blue dye (Sigma-Aldrich; Merck Millipore) was dissolved in normal saline $(30 \mathrm{mg} / \mathrm{ml})$, and was injected into the tail vein of rats ( $n=3 /$ group) for $10 \mathrm{sec}$ at a dose of $45 \mathrm{mg} / \mathrm{kg}$. After the dye had circulated for $120 \mathrm{~min}$, the chest cavity was opened and the left heart ventricle was cannulated. Each rat was perfused with $\mathrm{PBS}\left(37^{\circ} \mathrm{C}\right)$ for 2 min to clear the dye, ensuring the physiological pressure was maintained at $120 \mathrm{mmHg}$. Immediately after perfusion, the eyes were enucleated and retinas were carefully dissected. The weight of each retina was measured after thorough drying in a Speed-Vac. Albumin leakage into the retinal tissue was estimated via the measurement of extravasated Evans blue dye. Evans blue was extracted by incubating each retina in $0.3 \mathrm{ml}$ formamide for $18 \mathrm{~h}$ at $70^{\circ} \mathrm{C}$. The extract was filtered through a 30,000 MW filter at a speed of $300 \times \mathrm{g}$ for $45 \mathrm{~min}$ at $4^{\circ} \mathrm{C}$. The absorbance of the filtrate was measured using a spectrophotometer at 620 and $740 \mathrm{~nm}$, the absorption maximum for Evans blue in formamide. Calculations were based on the external standards dissolved in the same solvent. The concentration of dye in the extracts was calculated from a standard curve of Evans blue in formamide and normalized to the dry retinal weight and the time-averaged concentration of Evans Blue in the plasma.

Terminal deoxynucleotidyl transferase biotin-dUTP nick end labeling (TUNEL). Apoptosis was examined by TUNEL assay. TUNEL-positive nuclei in the GCL of the retina were counted. Briefly, following $8 \mathrm{~min}$ fixation with ice-cold acetone solution, cryopreserved tissue sections were washed three times with PBS. The sections were incubated with $1 \mathrm{ml}$ blocking buffer (3\% normal goat serum (Sigma-Aldrich; Merck Millipore) in PBS for $1 \mathrm{~h}$ at room temperature. Following incubation, the sections were washed in a permeabilization solution $(0.1 \%$ Triton X-100 in $0.1 \%$ sodium citrate) for $2 \mathrm{~min}$ on ice. After washing, the sections were incubated in $50 \mu 1$ TUNEL reaction mixture (cat. no. 12156792910; Roche Diagnostics GmbH, Mannheim, Germany) for $60 \mathrm{~min}$ at $37^{\circ} \mathrm{C}$ in the dark. Subsequently, the sections were counterstained with 4',6-diamidino-2-phenylindole. The sections were washed and observed under a fluorescence microscope (Olympus Corporation). The retinal cell numbers in the GCL were counted in the region within a 1-mm column.

Statistical analysis. Data are presented as the mean \pm standard deviation (each experiment was repeated 3 times) and were analyzed by SPSS 17.0 software (SPSS, Inc., Chicago, IL, USA). Results were analyzed by one-way analysis of variance followed by a least significant difference procedure. $\mathrm{P}<0.05$ was considered to indicate a statistically significant difference.

\section{Results}

Effects of Niaspan treatment on DR. To determine whether diabetes induces DR and whether Niaspan treatment regulates DR recovery, H\&E staining was performed. As presented in Fig. 1, non-diabetic rats exhibited normal retinas; all cell 
A

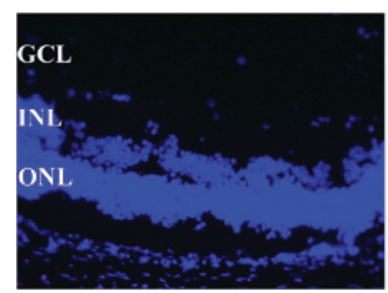

B
DR

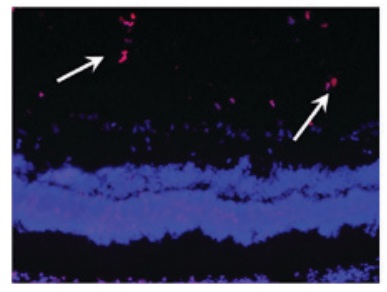

NA

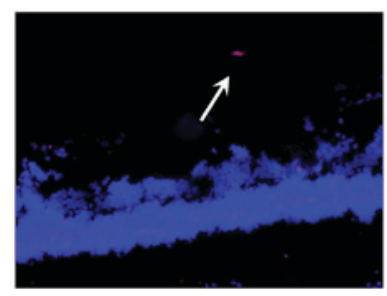

TUNEL+cells in RGCL

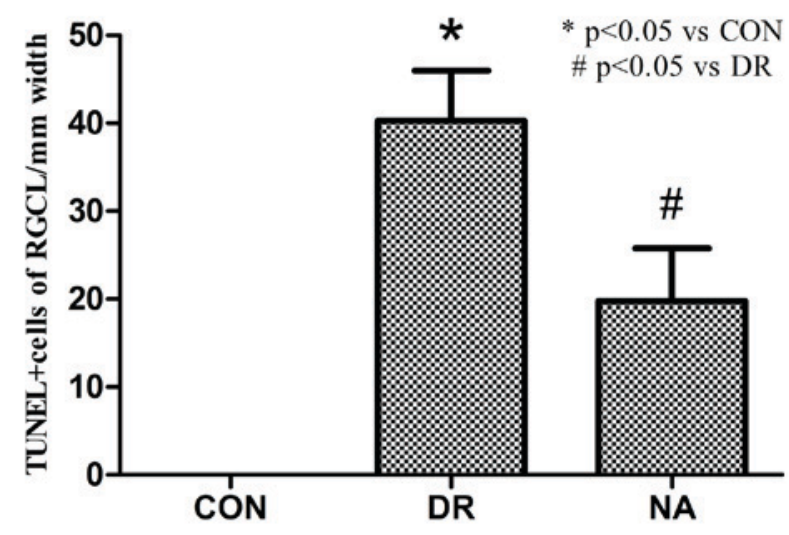

Figure 2. Detection of apoptosis in the RGCL of a DR model. (A) TUNEL staining of the retina ( $\mathrm{n}=4 /$ group). (B) Quantitative analysis of TUNEL ${ }^{+}$cells in the RGCL. The arrows indicate TUNEL ${ }^{+}$cells in the INL. "P $<0.05$ compared with nondiabetic retina in the CON group; "P $<0.05$ compared with diabetic retina in the DR group. CON, control; DR, diabetic retinopathy; NA, Niaspan; RGCL, retinal ganglion cell layer; INL, inner nuclear layer; ONL, outer nuclear layer.

layers of the retina were clear and neatly arranged. In the DR group, cells were disorganized after diabetic modeling. Obvious inflammatory cell infiltration in the GCL, hemorrhage, and neovascularization in the inner nuclear layer (INL) were observed. In the Niaspan-treated retina, retinal edema and hemorrhage were markedly attenuated, and the ganglionic layer was neatly arranged (Fig. 1A). Furthermore, cell number in the retinal GCL was significantly reduced in the DR group $(\mathrm{P}<0.05)$ compared with the control group. Treatment with Niaspan was able to significantly reverse the reduction in retinal cell numbers $(\mathrm{P}<0.05)$ compared with in the diabetic retinas (Fig. 1B).

Niaspan reduces DR-induced apoptosis of retinal cells in the GCL. Niaspan inhibits retinal cell apoptosis. Abundant numbers of $\mathrm{TUNEL}^{+}$cells were detected in the GCL of diabetic retina $(40.25 \pm 5.7373 ; \mathrm{P}<0.05)$ compared with in the control retina. Conversely, fewer apoptotic cells were detected in the GCL of the Niaspan-treated group (19.75 \pm 6.0208 ; $\mathrm{P}<0.05)$ compared with in the diabetic retina. No TUNEL ${ }^{+}$ cells were observed in the control group retinas (Fig. 2).

Niaspan prevents $D R$-induced BRB breakdown. DR-induced breakdown of the BRB was assessed by Evans blue extravasation from retinal vessels. As an initial approach, retinal blood vessel integrity was analyzed in flat mount retinas. Evans blue was observed as being confined to the retinal blood vessels without any leakage occurring in control rats. Conversely, the dye was shown to leak from the vessels to the surrounding tissue in DR rats. Niaspan treatment of diabetic rats was able to prevent this effect (Fig. 3A). Quantitative detection of Evans blue dye from the retinal tissue confirmed the results obtained by fluorescence microscopy. Diabetes increased BRB permeability in diabetic rats $(29.71 \pm 1.3214 \mathrm{ng}$ Evans blue/mg dry weight retina; $\mathrm{P}<0.05)$ compared with the control rats $(12.5 \pm 0.91591 \mathrm{ng}$ Evans blue/mg dry weight retina). Treatment with Niaspan significantly prevented BRB breakdown in diabetic rats $(18.15 \pm 0.45211 \mathrm{ng}$ Evans blue/mg dry weight retina; $\mathrm{P}<0.05)$ compared with untreated diabetic rats (Fig. 3B).

Niaspan reduces $D R$-induced $T N F-\alpha$ and $N F-\kappa B$ retinal expression. To determine whether treatment with Niaspan regulates TNF- $\alpha$ and NF- $\kappa \mathrm{B}$ expression TNF- $\alpha$ and NF- $\kappa \mathrm{B}$ expression levels were detected. As presented in Fig. 4, western blotting indicated that DR markedly increased the expression levels of TNF- $\alpha$ and NF- $\kappa$ B (Fig. 4A). A quantitative analysis revealed that there was a significant increase in TNF- $\alpha$ and $\mathrm{NF}-\kappa \mathrm{B}$ in diabetic rats $(\mathrm{P}<0.05)$ compared with in the control rats. Treatment with Niaspan was able to significantly prevent the increase in $\mathrm{TNF}-\alpha$ and $\mathrm{NF}-\kappa \mathrm{B}(\mathrm{P}<0.05)$ compared with in diabetic rats (Fig. 4B).

Immunohistochemistry indicated that treatment with Niaspan significantly decreased the expression levels of TNF- $\alpha$ (Fig. 5A). A quantitative analysis revealed that there was a significant increase in TNF- $\alpha$ expression in diabetic 
A
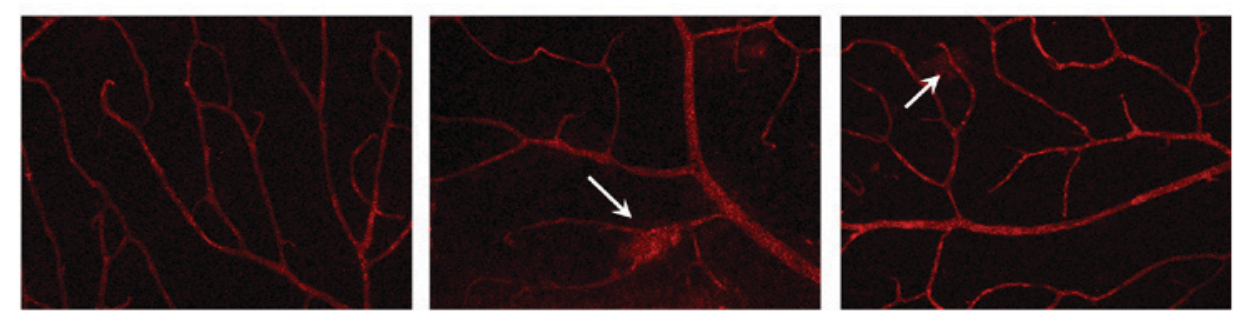

B

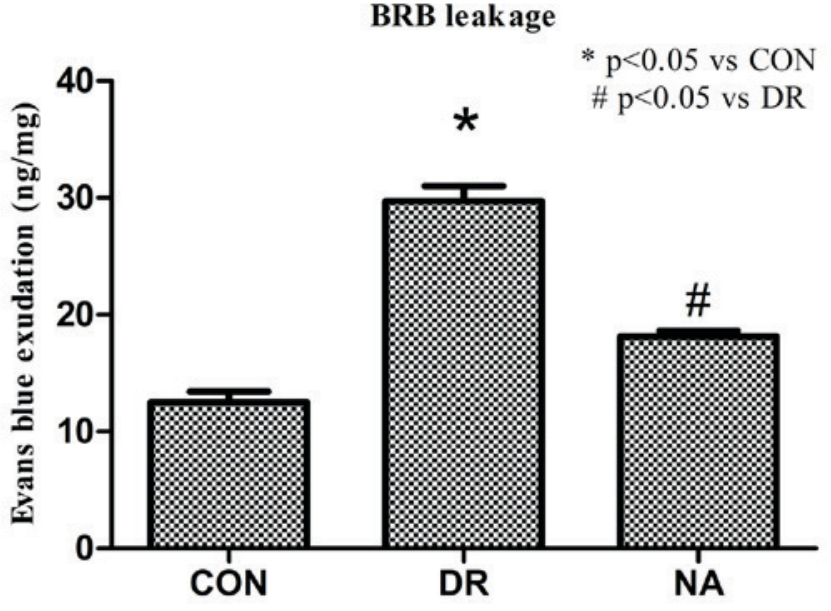

Figure 3. BRB leakage analysis in a DR model. (A) Evans blue assay for BRB leakage ( $n=4 /$ group). (B) Quantitative analysis of Evans blue exudation. The arrows indicate Evans blue extravasation from retinal vessels. $\mathrm{P}<0.05$ compared with nondiabetic retina in the CON group; ${ }^{\text {}} \mathrm{P}<0.05$ compared with diabetic retina in the DR group. CON, control; DR, diabetic retinopathy; NA, Niaspan; BRB, blood-retinal barrier.
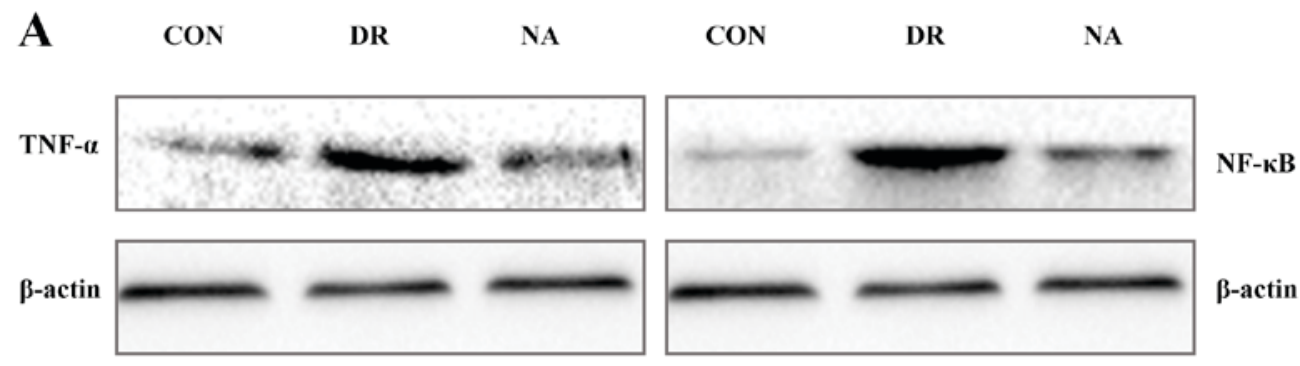

B Quantitative Data
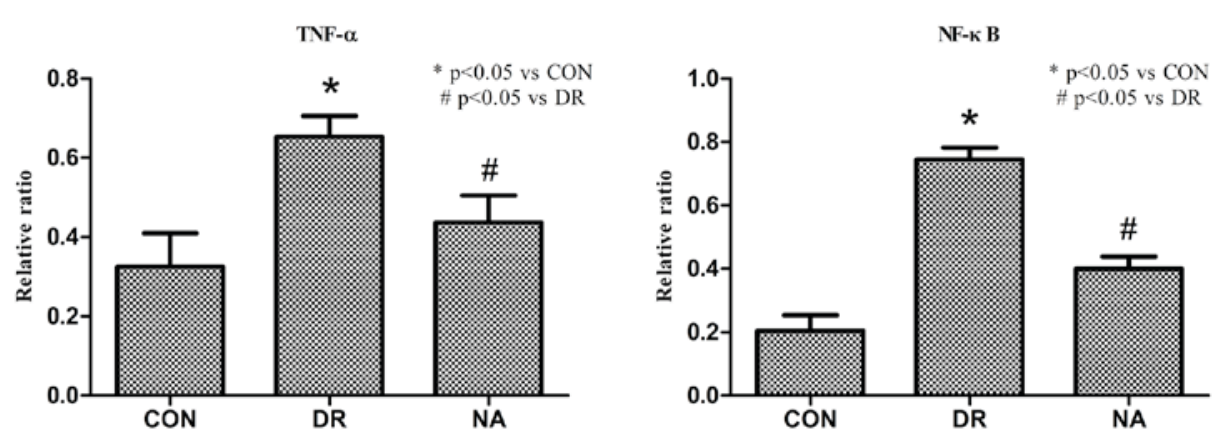

Figure 4. Effects of Niaspan on the retinal expression levels of TNF- $\alpha$ and NF- $\kappa$ B in a rat model of DR. (A) Western blotting and (B) quantitative analysis of TNF- $\alpha$ and NF- $\kappa$ B expression in the retina ( $n=4$ /group). ${ }^{*} \mathrm{P}<0.05$ compared with nondiabetic retina in the $\mathrm{CON}$ group; ${ }^{\#} \mathrm{P}<0.05$ compared with diabetic retina in the DR group. CON, control; DR, diabetic retinopathy; NA, Niaspan; TNF- $\alpha$, tumor necrosis factor- $\alpha$; NF- $\kappa$ B, nuclear factor- $\kappa$ B. 
A

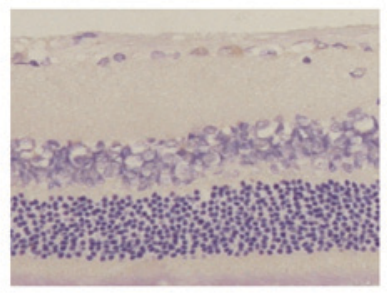

B

Quantitative Data
DR

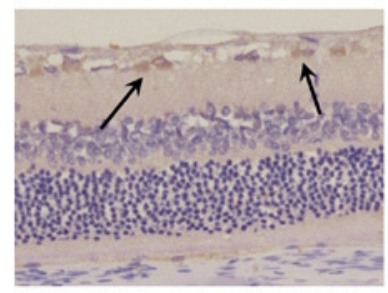

NA

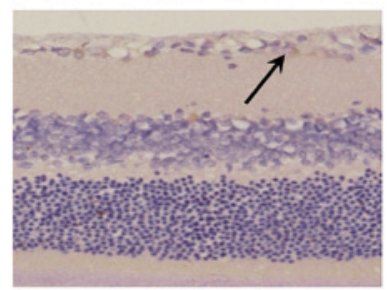

C
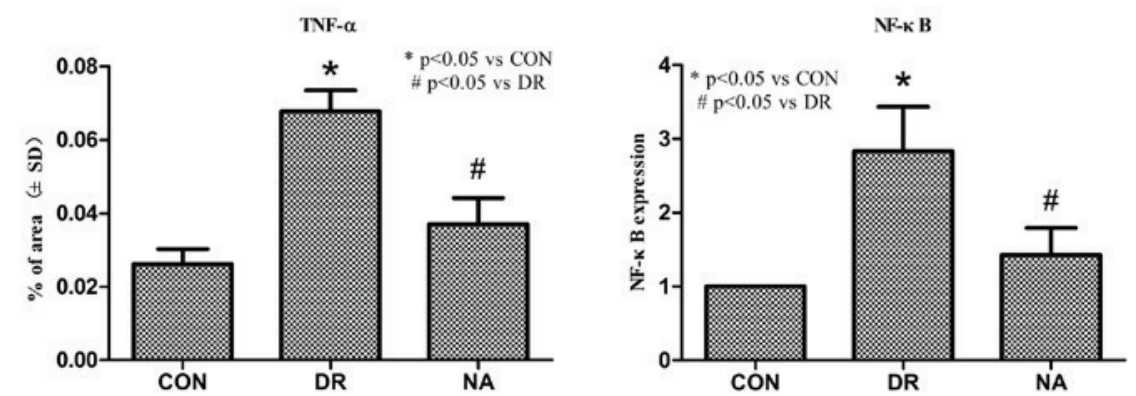

Figure 5. TNF- $\alpha$ and NF- $\mathrm{kB}$ expression in the retina of a DR model. (A) TNF- $\alpha$ immunostaining in the retina (n=7/group). (B) Quantitative analysis of TNF- $\alpha$ in control and diabetic retinas, and in the retina of Niaspan-treated rats ( $\mathrm{n}=7$ /group). (C) Reverse transcription-quantitative polymerase chain reaction was used to detect NF- $\mathrm{kB}$ expression in the retina. The arrows indicate TNF- $\alpha$ immunostaining in the retina. "P< $<05$ compared with nondiabetic retina in the CON group; ${ }^{\prime} \mathrm{P}<0.05$ compared with diabetic retina in the DR group. CON, control; DR, diabetic retinopathy; NA, Niaspan; TNF- $\alpha$, tumor necrosis factor- $\alpha$; $\mathrm{NF}-\kappa \mathrm{B}$, nuclear factor- $\mathrm{k}$.

rats $(\mathrm{P}<0.05)$ compared with control rats. Treatment with Niaspan was able to significantly prevent the increase in TNF- $\alpha(\mathrm{P}<0.05)$ compared with in diabetic rats (Fig. 5B). PCR analysis detected a significant increase in NF- $\kappa \mathrm{B}$ expression in diabetic rats $(\mathrm{P}<0.05)$ compared with control rats. Treatment with Niaspan was able to significantly prevent the increase in $\mathrm{NF}-\kappa \mathrm{B}$ expression $(\mathrm{P}<0.05)$ compared with in diabetic rats (Fig. 5C).

Niaspan reduces DR-induced iNOS and ICAM-1 retinal expression. To determine whether Niaspan treatment regulates iNOS and ICAM-1 target gene expression ICAM-1 and iNOS expression levels were detected. As presented in Fig. 6, western blotting indicated that Niaspan treatment markedly decreased the expression levels of ICAM-1 and iNOS (Fig. 6A). A quantitative analysis revealed that there was a significant increase in iNOS and ICAM-1 expression in diabetic rats $(\mathrm{P}<0.05)$ compared with in control rats. Treatment with Niaspan was able to significantly prevent the increase in iNOS and ICAM-1 expression $(\mathrm{P}<0.05)$ compared with in diabetic rats (Fig. 6B). PCR analysis detected a significant increase in iNOS and ICAM-1 expression in diabetic rats $(\mathrm{P}<0.05)$ compared with in control rats. Treatment with Niaspan was able to significantly prevent the increase in iNOS and ICAM-1 $(\mathrm{P}<0.05)$ expression compared with in diabetic rats (Fig. 6C).

\section{Discussion}

The present study used the common animal model of STZ-induced DR; the results confirmed that STZ injection resulted in diabetes and significantly induced DR; however, long-term Niaspan treatment reduced the formation and development of DR, and inhibited the development of inflammation. This conclusion is based on several lines of experimental evidence. Initially, the present study indicated that treatment of diabetic rats with Niaspan markedly decreased hemorrhage, leukocyte infiltration and apoptosis in the GCL of the diabetic retina. STZ has previously been demonstrated to induce hyperglycemia and lead to the generation of oxidative stress (11), which is a typical characteristic of DR in rats, which may promote the destruction of endothelial integrity and breakdown of the BRB. These alterations in endothelial integrity were detected by cell apoptosis analysis and retinal vascular permeability assay. Thirdly, treatment of DR with Niaspan significantly decreased the expression of inflammatory mediators, including TNF- $\alpha$, NF- $\kappa \mathrm{B}$, iNOS and ICAM-1 compared with in diabetic retinas.

DR is a vascular, neuroinflammatory disease that is characterized by cell apoptosis and neuroinflammation. Apoptosis, which is a type of programmed cell death, has been detected in the retina following ischemia-induced injury. A previous study reported that during the course of diabetes, apoptosis occurs early in endothelial cells and retinal ganglion cells (12). In addition, endothelial cell and pericyte loss is one of the earliest and key manifestations of DR, which may lead to BRB breakdown (13). An early sign of DR in an experimental model of diabetes is vascular inflammation due to oxidative stress; furthermore, proinflammatory cytokines (14) and inflammatory mediators have been reported to promote increased vascular permeability, leukocyte adhesion and retinal cell 
A CON DR NA CON DR NA
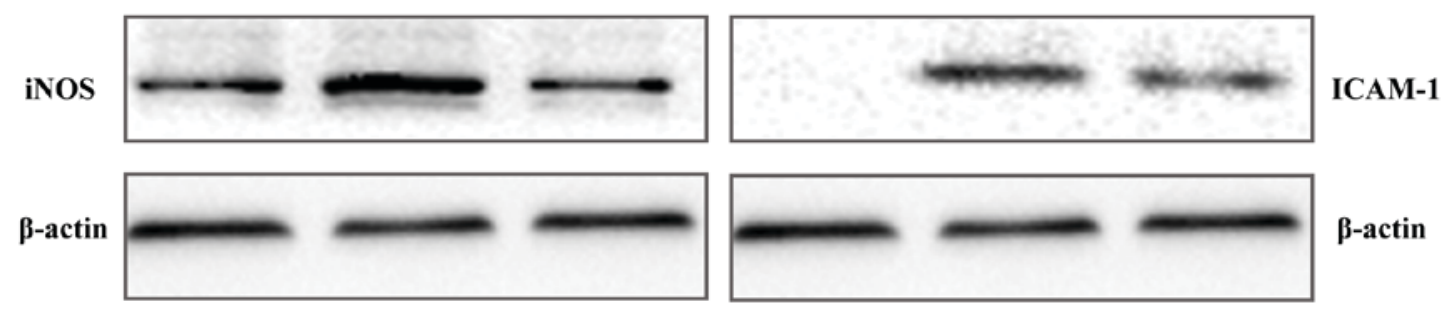

B Quantitative Data
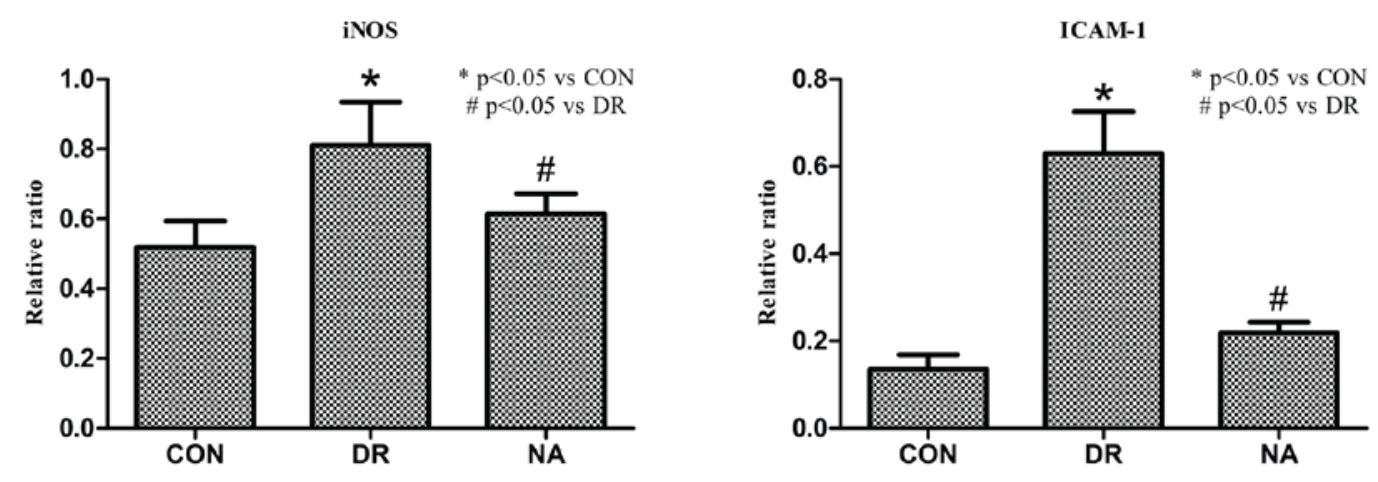

C

iNOS
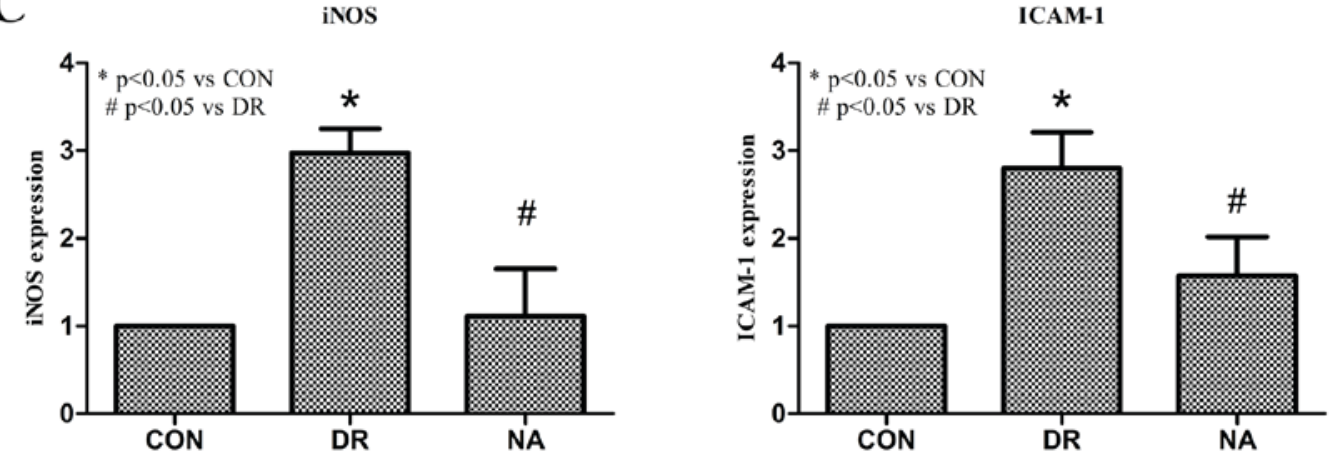

Figure 6. Effects of Niaspan on the expression levels of iNOS and ICAM-1 in the retina of a rat model of DR. (A) Western blotting and (B) quantitative analysis of iNOS and ICAM-1 expression in the retina ( $\mathrm{n}=4$ /group). (C) Reverse transcription-quantitative polymerase chain reaction analysis detected iNOS and ICAM-1 expression in the retina. ${ }^{*} \mathrm{P}<0.05$ compared with nondiabetic retina in the CON group; ${ }^{*} \mathrm{P}<0.05$ compared with diabetic retina in the DR group. CON, control; DR, diabetic retinopathy; NA, Niaspan; iNOS, inducible nitric oxide synthase; ICAM-1, intercellular adhesion molecule-1.

death (15). As a consequence, Niaspan may mitigate cell apoptosis and BRB breakdown by downregulating inflammatory factors.

TNF- $\alpha$ is a major proinflammatory cytokine that is involved in numerous inflammatory pathologies, and is predominantly produced by macrophages (16). Increased levels of TNF- $\alpha$ have been detected in the vitreous of diabetic patients with proliferative DR (17) and in diabetic rat retinas (18). TNF- $\alpha$ is a potent mediator of leukostasis induced by vascular endothelial growth factor, interleukin-1 $\alpha$, and platelet-activating factor in the retinal vasculature (19), and also mediates the cell death/apoptosis of retinal neurons and vascular endothelial cells in DR (13). The involvement of the inflammatory cytokine TNF- $\alpha$ in the apoptotic cell death of retinal endothelial cells during the early and late stages of
DR in a rat model of STZ-induced diabetes has previously been investigated $(18,20)$. The present study suggested that Niaspan markedly decreases TNF- $\alpha$ in the diabetic retina, which may contribute to the beneficial effects of Niaspan treatment.

Activation of NF- $\mathrm{kB}$ induces the expression of numerous inflammatory cytokines, including TNF- $\alpha$ (21), which are crucial factors in inflammation. However, TNF- $\alpha$ is not only induced by NF- $\kappa B$, but is also a strong activator of NF- $\kappa \mathrm{B}(22)$. In addition, inhibition of TNF- $\alpha$ may inhibit the activity of NF- $\mathrm{KB}$ (23), which is a widely expressed inducible transcription factor that is an important regulator of several genes involved in mammalian inflammatory and immune responses, proliferation and apoptosis (24). The present study demonstrated that Niaspan may significantly prevent the increase in TNF- $\alpha$ and NF-kB expression, which was induced by DR. 
These findings suggested that $\mathrm{NF}-\kappa \mathrm{B}$, under the regulation of TNF- $\alpha$, may be associated with diabetes-induced inflammation in the retina.

Upregulation of iNOS has been detected in the retinas of experimental diabetic rodents and human patients in previous studies $(25,26)$. In addition, iNOS serves an important role in leukostasis, apoptosis and BRB breakdown $(27,28)$. Concurrently, white blood cells interact with, and bind to, ICAM-1 on the surface of endothelial cells in a multi-step process leading to adherence of the blood cells to the endothelial wall. Notably, suppression of ICAM-1 attenuates retinal leukostasis in animal models of DR (29). TNF- $\alpha$ regulates the expression of adhesion molecules, including ICAM-1, which is correlated with the increase in leukostasis and BRB breakdown in diabetic rat retinas (18). Furthermore, a previous study demonstrated that suppression of $\mathrm{NF}-\kappa \mathrm{B}$ (11 activation in the retinas of diabetic rats inhibited the expression of inflammatory mediators, including iNOS and ICAM-1, and capillary degeneration and pericyte loss in these animals (24).

In conclusion, the present study indicated that DR leads to the generation of important inflammatory cytokines, which may lead to endothelial cell apoptosis and BRB breakdown. These findings suggested that Niaspan-induced downregulation of TNF- $\alpha$ may contribute to amelioration of the inflammatory reaction in diabetic rats. Furthermore, $\mathrm{TNF}-\alpha$ may induce reactive oxygen species formation, $\mathrm{NF}-\kappa \mathrm{B}$ activation and iNOS expression in inflammatory cells, and rapidly upregulate the expression of ICAM-1 at the endothelial surface (30). In accordance with these findings, the reduction of TNF- $\alpha$ may reduce apoptosis of endothelial cells and BRB breakdown. The results of the present study strongly indicated that Niaspan may be considered a potential therapeutic agent for the treatment of DR via inhibition of the inflammatory process.

\section{Acknowledgements}

The present study was supported by the National Science Foundation of Tianjin (grant nos. 12JCYBJC33900, 14JCYBJC28000 and 2013KZ119) and the National Natural Science Foundation of China (grant nos. 81371038 and 91442124).

\section{References}

1. Aiello LP, Gardner TW, King GL, Blankenship G, Cavallerano JD, Ferris FL III and Klein R: Diabetic retinopathy. Diabetes Care 21: 143-156, 1998.

2. Stem MS and Gardner TW: Neurodegeneration in the pathogenesis of diabetic retinopathy: Molecular mechanisms and therapeutic implications. Curr Med Chem 20: 3241-3250, 2013.

3. Abcouwer SF: Angiogenic factors and cytokines in diabetic retinopathy. J Clin Cell Immunol (Suppl 1), 2013.

4. Chapman MJ, Assmann G, Fruchart JC, Shepherd J and Sirtori C; European Consensus Panel on HDL-C: Raising high-density lipoprotein cholesterol with reduction of cardiovascular risk: The role of nicotinic acid-a position paper developed by the European Consensus Panel on HDL-C. Curr Med Res Opin 20: 1253-1268, 2004

5. Elam MB, Hunninghake DB, Davis KB, Garg R, Johnson C, Egan D, Kostis JB, Sheps DS and Brinton EA: Effect of niacin on lipid and lipoprotein levels and glycemic control in patients with diabetes and peripheral arterial disease: The ADMIT study: A randomized trial. Arterial Disease Multiple Intervention Trial. JAMA 284: 1263-1270, 2000.
6. Heemskerk MM, Dharuri HK, van den Berg SA, Jónasdóttir HS, Kloos DP, Giera M, van Dijk KW and van Harmelen V: Prolonged niacin treatment leads to increased adipose tissue PUFA synthesis and anti-inflammatory lipid and oxylipin plasma profile. J Lipid Res 55: 2532-2540, 2014.

7. Si Y, Zhang Y, Zhao J, Guo S, Zhai L, Yao S, Sang H, Yang N, Song G, Gu J and Qin S: Niacin inhibits vascular inflammation via downregulating nuclear transcription factor- $\kappa \mathrm{B}$ signaling pathway. Mediators Inflamm 2014: 263786, 2014

8. Zhang W and Yan H: Simvastatin increases circulating endothelial progenitor cells and reduces the formation and progression of diabetic retinopathy in rats. Exp Eye Res 105: 1-8, 2012.

9. Lei S, Li H, Xu J, Liu Y, Gao X, Wang J, Ng KF, Lau WB, Ma XL, Rodrigues $\mathrm{B}$, et al: Hyperglycemia-induced protein kinase $\mathrm{C} \beta 2$ activation induces diastolic cardiac dysfunction in diabetic rats by impairing caveolin-3 expression and Akt/eNOS signaling. Diabetes 62: 2318-2328, 2013.

10. Livak KJ and Schmittgen TD: Analysis of relative gene expression data using real-time quantitative PCR and the 2(-Delta Delta C (T)) Method. Methods 25: 402-408, 2001.

11. Arden GB and Sivaprasad S: The pathogenesis of early retinal changes of diabetic retinopathy. Doc Ophthalmol 124: 15-26, 2012.

12. Barber AJ, Lieth E, Khin SA, Antonetti DA, Buchanan AG and Gardner TW: Neural apoptosis in the retina during experimental and human diabetes. Early onset and effect of insulin. J Clin Invest 102: 783-791, 1998.

13. Joussen AM, Doehmen S, Le ML, Koizumi K, Radetzky S, Krohne TU, Poulaki V, Semkova I and Kociok N: TNF-alpha mediated apoptosis plays an important role in the development of early diabetic retinopathy and long-term histopathological alterations. Mol Vis 15: 1418-1428, 2009.

14. Joussen AM, Poulaki V, Le ML, Koizumi K, Esser C, Janicki H, Schraermeyer U, Kociok N, Fauser S, Kirchhof B, et al: A central role for inflammation in the pathogenesis of diabetic retinopathy. FASEB J 18: 1450-1452, 2004.

15. Krady JK, Basu A, Allen CM, Xu Y, LaNoue KF, Gardner TW and Levison SW: Minocycline reduces proinflammatory cytokine expression, microglial activation, and caspase-3 activation in a rodent model of diabetic retinopathy. Diabetes 54: 1559-1565, 2005.

16. Mukhopadhyay S, Hoidal JR and Mukherjee TK: Role of TNFalpha in pulmonary pathophysiology. Respir Res 7: 125, 2006.

17. Abu el Asrar AM, Maimone D, Morse PH, Gregory S and Reder AT: Cytokines in the vitreous of patients with proliferative diabetic retinopathy. Am J Ophthalmol 114: 731-736, 1992.

18. Joussen AM, Poulaki V, Mitsiades N, Kirchhof B, Koizumi K, Döhmen S and Adamis AP: Nonsteroidal anti-inflammatory drugs prevent early diabetic retinopathy via TNF-alpha suppression. FASEB J 16: 438-440, 2002.

19. Vinores SA, Xiao WH, Shen J and Campochiaro PA: TNF-alpha is critical for ischemia-induced leukostasis, but not retinal neovascularization nor VEGF-induced leakage. J Neuroimmunol 182: 73-79, 2007.

20. Jiang Y, Zhang Q, Soderland C and Steinle JJ: TNFa and SOCS3 regulate IRS-1 to increase retinal endothelial cell apoptosis. Cell Signal 24: 1086-1092, 2012.

21. Sun X, Han F, Yi J, Han L and Wang B: Effect of aspirin on the expression of hepatocyte NF- $\kappa B$ and serum TNF- $\alpha$ in streptozotocin-induced type 2 diabetic rats. J Korean Med Sci 26: 765-770, 2011.

22. Takeda K, Kermani P, Anastasia A, Obinata Y, Hempstead BL and Kurihara H: BDNF protects human vascular endothelial cells from $\mathrm{TNF} \alpha-$ induced apoptosis. Biochem Cell Biol 91: 341-349, 2013.

23. Gao JJ, Hu YW, Wang YC, Sha YH, Ma X, Li SF, Zhao JY, Lu JB, Huang C, Zhao JJ, et al: ApoM suppresses TNF- $\alpha$-induced expression of ICAM-1 and VCAM-1 through inhibiting the activity of NF-кB. DNA Cell Biol 34: 550-556, 2015.

24. Kern TS: Contributions of inflammatory processes to the development of the early stages of diabetic retinopathy. Exp Diabetes Res 2007: 95103, 2007.

25. Ellis EA, Guberski DL, Hutson B and Grant MB: Time course of NADH oxidase, inducible nitric oxide synthase and peroxynitrite in diabetic retinopathy in the BBZ/WOR rat. Nitric Oxide 6: 295-304, 2002.

26. Abu El-Asrar AM, Desmet S, Meersschaert A, Dralands L, Missotten L and Geboes K: Expression of the inducible isoform of nitric oxide synthase in the retinas of human subjects with diabetes mellitus. Am J Ophthalmol 132: 551-556, 2001. 
27. Leal EC, Manivannan A, Hosoya K, Terasaki T, Cunha-Vaz J, Ambrósio AF and Forrester JV: Inducible nitric oxide synthase isoform is a key mediator of leukostasis and blood-retinal barrier breakdown in diabetic retinopathy. Invest Ophthalmol Vis Sci 48: 5257-5265, 2007.

28. Rosales MA, Silva KC, Duarte DA, de Oliveira MG, de Souza GF, Catharino RR, Ferreira MS, Lopes de Faria JB and Lopes de Faria JM: S-nitrosoglutathione inhibits inducible nitric oxide synthase upregulation by redox posttranslational modification in experimental diabetic retinopathy. Invest Ophthalmol Vis Sci 55: 2921-2932, 2014
29. Miyamoto K, Khosrof S, Bursell SE, Rohan R, Murata T, Clermont AC, Aiello LP, Ogura Y and Adamis AP: Prevention of leukostasis and vascular leakage in streptozotocin-induced diabetic retinopathy via intercellular adhesion molecule-1 inhibition. Proc Natl Acad Sci USA 96: 10836-10841, 1999.

30. Zhang W, Liu H, Al-Shabrawey M, Caldwell RW and Caldwell RB: Inflammation and diabetic retinal microvascular complications. J Cardiovasc Dis Res 2: 96-103, 2011. 